\title{
Continuation of Partial-Wave Two-Cluster Atomic Scattering Amplitudes ${ }^{\star}$
}

\author{
J. Nuttall and S. R. Singh \\ Department of Physics University of Western Ontario London, Ontario, Canada
}

\begin{abstract}
It is shown, with some restrictions, that on-shell two-cluster partialwave scattering amplitudes for atomic systems whose particles interact via two-body Coulomb potentials have analytic continuations in the complex energy plane below the physical part of the real axis. The result is proved only for energies lower than any three (or more) cluster threshold. Poles of the amplitudes can occur only at discrete eigenvalues of the rotated Hamiltonian which may be reached by continuation along the same path. The method of proof uses analyticity related to a generalized scaling transformation and the boost transformation.
\end{abstract}

\section{Introduction}

In a previous paper [1], referred to as I, we studied an $N$-particle atomic system whose Hamiltonian with center of mass part removed is $H$. We concentrated on the range of energy below $E_{\min }$ the lowest threshold of all those corresponding to three or more bound clusters, so that only channels consisting of two bound clusters are open, and, to simplify the argument, also assumed that at least one of each pair of clusters was neutral. We proved I Theorem 1 which showed that the limit

$$
T_{f i}(E)=\lim _{\varepsilon \downarrow 0}\left(\psi^{f}, V^{f} G(E+i \varepsilon) V^{i} \psi^{i}\right)
$$

exists for $E$ in the above range on the complement of a closed set of measure zero. In (1) $\psi^{i}, \psi^{f}$ describe the two-cluster states in channels $i, f$ respectively and each correspond to a given partial wave, and $V^{i}, V^{f}$ are respectively the sums of the two-body potentials between all pairs of particles in different clusters of $i, f$. We also use $G(z)=(Z-H)^{-1}$.

The effect of I was thus to show that, under the above conditions, the conventional time-independent formula for the scattering amplitude, $T_{f i}(E)+$ Born term, makes sense. In this paper we show that $T_{f i}(E)$ (and thus the full amplitude) may be

\footnotetext{
* Supported in part by the National Research Council of Canada
} 
analytically continued to $T_{f i}(z)$, a function meromorphic in $z$ in some neighborhood of any part of the real axis between two-cluster thresholds and below threecluster thresholds. Poles can occur only at values of $z$ corresponding to discrete eigenvalues of the "rotated" Hamiltonian, for suitable rotations. The result is stated as Theorem 1 below, and is proved under conditions (i) to (iii) below, the third being added merely to reduce the complexity of the exposition.

(i) The system is described by the usual non-relativistic Hamiltonian with a Coulomb potential $V$ acting between each pair of particles.

(ii) For every channel corresponding to two-bound clusters that is open below $E_{\min }$ at least one cluster is neutral.

(iii) Each bound cluster referred to in (ii) has angular momentum zero. As in $I$, the class of potentials for which the results hold is larger than the Coulombic of (i).

Other work aimed in the same direction as this has recently been reported by Combes [2] and Tip [3]. Similar results have been obtained independently by Hagedorn [4] but with the crucial restriction to potentials with exponential decay at infinity.

The method of proof is based on the algebraic manipulations of I, which relate the amplitude to the solution of a coupled-channel integral equation. The integration contours are then distorted as in $[5,6]$. With Coulomb potentials it is necessary for the initial and final momenta (complex) to remain on their respective contours.

We deduce the needed analyticity of the effective potentials appearing in the integral equation by making use of an analytic family of operators related to the Hamiltonian by a set of transformations involving the boost group and a generalized scale transformation, described in Sect. 2. In a previous summary of this work [7] a different, less powerful method of deducing analyticity was outlined.

The analyticity of the off-shell amplitude in several complex variables is used to deduce the analyticity of $T_{f i}(z)$ and to obtain the connection between the poles of $T_{f i}(z)$ and the eigenvalues of the rotated Hamiltonian. In this way we demonstrate that, even if the integral equations that we use, such as the WeinbergVan Winter equation, have spurious homogeneous solutions, not related to eigenvalues of the rotated Hamiltonian, then $T_{f i}(z)$ does not have corresponding poles.

An incidental result of our work is that I Theorem 1 may be strengthened in that the limit may be shown to exist for all but a discrete set of points, whose only limit points may be thresholds.

The result of this paper tends to justify a number of calculations of resonance positions in atomic systems which have been performed by searching for eigenvalues of the rotated Hamiltonian [8].

Before presenting the details of the proof we remind the reader of the definitions of some of the symbols in I that will be used here. For further details see I.

We let $D_{2}^{m}, m=1, \ldots$, be the two-cluster decompositions $\left(C_{1}, C_{2}\right)$ of the Nparticle system. For each $m$ the Hilbert space $\mathscr{H}=L^{2}\left(\mathbb{R}^{3 N-3}\right)$ for the whole system may be decomposed as

$$
\mathscr{H}=\mathscr{H}_{m}^{(1)} \otimes \mathscr{H}_{m}^{(2)} \otimes \mathscr{H}_{m}^{(1,2)}
$$


where $\mathscr{H}_{m}^{(1)}, \mathscr{H}_{m}^{(2)}$ are the state spaces for the internal motion of the particles in clusters $C_{1}, C_{2}$ respectively, and $\mathscr{H}_{m}^{(1,2)}$ is the state space for the relative motion of the two clusters. There is a corresponding decomposition of configuration space. If $X \in \mathbb{R}^{3 N-3}$, we set

$$
X=\left(s_{m}^{1}, s_{m}^{2}, \mathbf{r}_{m}\right)
$$

for each $m$, so that $s_{m}^{1}, s_{m}^{2}$ are respectively internal coordinates of $C_{1}, C_{2}$ and $\mathbf{r}_{m} \in \mathbb{R}^{3}$ is the vector joining the centers of mass of the two clusters. We may write $s_{m}^{1}=\left(\mathbf{s}_{m 1}^{1}, \mathbf{s}_{m 2}^{1}, \ldots\right), \mathbf{s}_{m k}^{1} \in \mathbb{R}^{3}$ and similarly for $s_{m}^{2}$.

Those decompositions $D_{2}^{m}$ which correspond to the channels of condition (ii) are labelled $m=1, \ldots, j$ (not $j_{0}$ as in $I$ ).

The operator $V_{D_{2}^{n} D_{1}}$ is the sum of all two-body potentials acting between pairs of particles in different clusters of $D_{2}^{m}$.

The operator $\bar{I}(z)$ is obtained from the kernel of the Weinberg-Van Winter equation by removing those terms which are singular when $z$ takes on real values below $E_{\min }$ and above the lowest two-cluster threshold.

\section{Transformations and Analytic Families}

We extend the ideas of Combes and Thomas [9] by introducing a set of unitary transformations on $\mathscr{H}$ given by (using the notation of [9])

$$
(u(\tau, \zeta) \phi)(X)=(\operatorname{det} \zeta)^{(N-1) / 2} e^{-i\langle X, \zeta \tau\rangle} \phi(\zeta X), \phi \in \mathscr{H}
$$

where $\tau \in \mathbb{R}^{3(N-1)}$ and $\zeta$ is a $3 \times 3$ real matrix. For $X$ as in (2) we define $\zeta X=$ $\left(\zeta \mathbf{s}_{m 1}^{1}, \ldots, \zeta \mathbf{s}_{m 1}^{2}, \ldots, \zeta \mathbf{r}_{m}\right)$.

In the momentum representation

$$
(\widetilde{u(\tau, \zeta) \phi})(P)=(\operatorname{det} \zeta)^{-(N-1) / 2} \tilde{\phi}\left(\zeta^{-1} P+\bar{M} \tau\right), \phi \in \mathscr{H}
$$

We have

$$
u(\tau, \zeta) u\left(\tau^{\prime}, \zeta^{\prime}\right)=u\left((\tau, \zeta) \times\left(\tau^{\prime}, \zeta^{\prime}\right)\right)=u\left(\zeta^{-1} \tau+\tau^{\prime}, \zeta \zeta^{\prime}\right)
$$

Just as in [9], we construct an analytic family $H(\gamma)$, where now $\gamma=(\tau, \zeta)$, $\tau \in \mathbb{C}^{3(N-1)}, \zeta$ a $3 \times 3$ complex matrix. (Note that $\gamma$ is a generalization of that used in I). Analyticity of $V_{i j}(\gamma)$ will follow so long as $\operatorname{Re}\left(\zeta^{T} \zeta\right)$ is positive definite. The set of $\gamma$ for which $H(\gamma)$ is analytic we call $Y$. We have

$$
H\left(\gamma \times \gamma_{0}\right)=u(\gamma) H\left(\gamma_{0}\right) u^{-1}(\gamma)
$$

for $\gamma$ real and $\gamma, \gamma_{0}, \gamma \times \gamma_{0} \in \Upsilon$. The operator $H(0,1)$ is the Hamiltonian of the system.

The properties of the spectrum are analogous to the case studied in [9]. The only difference is the spectra of $H_{0}^{D}(\gamma), H_{0}^{C}(\gamma)$ which are the sets of values taken by $K_{D}\left(\zeta^{-1} P+\bar{M} \tau\right), K_{C}\left(\zeta^{-1} P+\bar{M} \tau\right)$. We shall not trouble to describe $\sigma_{e}(\gamma)$ exactly, but we assert that, if $B \subset \mathbb{C}$ is a given bounded set, then $\sigma_{e}(\gamma) \cap B \rightarrow \sigma_{e}\left(\gamma_{z}\right) \cap B$ as $\gamma \rightarrow \gamma_{z}$, where $\gamma_{z}=(\tau, 1 z), z \in \mathbb{C}$. Moreover, that part of the discrete spectrum of $H(\gamma)$ not contained in $\sigma_{e}(\gamma)$ is independent of $\gamma$, and is the same as that discussed by Combes and Thomas for the case $\gamma=\gamma_{z}$. With each $E \in \sigma_{d}(\gamma), E \notin \sigma_{e}(\gamma)$, is an analytic 
vector $\psi(\gamma)$ satisfying

$$
H(\gamma) \psi(\gamma)=E \psi(\gamma)
$$

for suitable $\gamma$.

For a cluster decomposition $D_{2}^{m}$ we define

$$
\begin{aligned}
& u_{m}(\gamma)=u^{D}(\gamma) \\
& \bar{u}_{m}(\gamma)=u^{C_{1}}(\gamma) \otimes u^{C_{2}}(\gamma)
\end{aligned}
$$

where the operators on the right are analogous to those in [9], eq. (3). Thus we have

$$
u(\gamma)=u_{m}(\gamma) \otimes \bar{u}_{m}(\gamma)
$$

where $u_{r, l}(\gamma), \bar{u}_{m}(\gamma)$ act in $\mathscr{H}_{m}^{(1,2)}, \mathscr{H}_{m}^{(1)} \otimes \mathscr{H}_{m}^{(2)}$ respectively.

Repetition of the line of reasoning taken in I leads to the following result.

Lemma 1. There exist sets $S_{1} \subset \mathbb{C}, S_{2} \subset Y$ such that for $z \in S_{1}, \gamma \in S_{2}$, there is a family $M(z ; \gamma)$ analytic in $z, \gamma$ except for a finite set of points $S_{1}^{d} \subset S_{1}$ at which $M(z ; \gamma)$ may have a pole. We have, for $\gamma$ real, $\gamma, \gamma_{0}, \gamma \times \gamma_{0} \in S_{2}$

$$
M\left(z ; \gamma \times \gamma_{0}\right)=u(\gamma) M\left(z ; \gamma_{0}\right) u^{-1}(\gamma)
$$

and

$$
M(z ; 0,1)=(1-\bar{I}(z))^{-1} \bar{I}(z) .
$$

Also, for each $m=1, \ldots, j$ there exists $\phi_{m}(\gamma) \in \mathscr{H}_{m}^{(1)} \otimes \mathscr{H}_{m}^{(2)}$, analytic in $\gamma$, such that for real $\gamma$

$$
\bar{u}_{m}(\gamma) \phi_{m}=\phi_{m}(\gamma) .
$$

In addition, there exists $\mu>0$ such that, with $\bar{\phi}_{m}=e^{\mu\left|s_{m}\right|} \phi_{m}$, we have $\bar{\phi}_{m}(\gamma) \in \mathscr{H}_{m}^{(1)} \otimes$ $\mathscr{H}_{m}^{(2)}$ analytic in $\gamma$, and for real $\gamma$

$$
\bar{u}_{m}(\gamma) \bar{\phi}_{m}=\bar{\phi}_{m}(\gamma) \text {. }
$$

For $S_{1}$ we may take $S_{1}=\left\{z: b_{r}<\operatorname{Re} z<E_{0},|\operatorname{Imz}|<h_{i}\right\}$, where $b_{j}<b_{r}<$ $E_{0}<E_{\min }$ and $h_{i}>0$ is suitably small. For $S_{2}$ we may choose $\{\gamma=(\tau, \zeta): \exists \lambda$, $|\arg \lambda|<\alpha_{0}$ with $\left.\|\zeta-\lambda 1\|<\delta_{1},\|\operatorname{Im} \zeta \tau\|<\delta_{2}\right\}$ where $\alpha_{0}, \delta_{1}, \delta_{2}>0$ depend on $S_{1}$.

In I we introduced the bounded operator $B_{n m}(z)$ which was a factor in the kernel of our integral equation. With $f \in \mathscr{H}_{m}^{(1,2)}, f^{\prime} \in \mathscr{H}_{n}^{(1,2)}$ we have

$$
\left(f^{\prime}, B_{n m}(z) f\right)=\left(\left(\bar{\phi} \otimes f^{\prime}\right), \beta_{n m}\left(\phi_{m} \otimes f\right)\right)
$$

where $\beta_{n m}: \mathscr{H} \rightarrow \mathscr{H}$ is given by

$$
\beta_{n m}=\left(e^{-\mu\left|s_{n}\right|} \otimes \omega_{n}\right) V_{D_{2}^{n} D_{1}}\left(1 \otimes \omega_{m}\right)\left[1+\left(1 \otimes \omega_{m}^{-1}\right) M(z ; 0,1)\left(1 \otimes \omega_{m}\right)\right] .
$$

For future convenience in this paper, we shall use for all $m$

$$
\omega_{m}^{-1}(r)=\omega^{-1}(r)=r^{-1}\left(1-e^{-r}\right)
$$

Lemma 2. There is a family of bounded operators $B_{n m}(z ; \gamma): \mathscr{H}_{m}^{(1,2)} \rightarrow \mathscr{H}^{(1,2)}$, meromorphic in $S_{1} \otimes S_{2}$ with poles only at $z \in S_{1}^{d}$, such that, for $\gamma$ real, $\gamma, \gamma_{0}, \gamma \times \gamma_{0} \in S_{2}$,

$$
B_{n m}\left(z ; \gamma \times \gamma_{0}\right)=u_{n}(\gamma) B_{n m}\left(z ; \gamma_{0}\right) u_{m}^{-1}(\gamma)
$$


and

$$
B_{n m}(z ; 0,1)=B_{n m}(z)
$$

Proof. The operator $\beta_{n m}$ has an extension to $\beta_{n m}(\gamma)$ since the factors $\left(e^{-\mu\left|s_{n}\right|} \otimes\right.$ $\left.\omega_{n}\right) V_{D_{2}^{n} D_{1}}\left(1 \otimes \omega_{m}\right)$ and $\left(1 \otimes \omega_{m}^{-1}\right) M(z ; 0,1)\left(1 \otimes \omega_{n}\right)$ have extensions. In the first case this is obvious from the definition of $u(\gamma)$ and Lemma 7 of I. In the second case, we have

$$
M(z ; \zeta)=u^{-1}\left(\tau^{\prime}, 1\right) M\left(z ; \zeta^{-1} \tau^{\prime}+\tau, \zeta\right) u\left(\tau^{\prime}, 1\right)
$$

which means that for each fixed $\tau, \zeta, M(z ; \tau, \zeta)$ has a boost analytic extension. Thus the argument of Lemma 5 of I may be used to show that $M(z ; \gamma)$ is pseudolocal, from which it follows that the extension of $\left(1 \otimes \omega_{m}^{-1}\right) M(z ; 0,1)\left(1 \otimes \omega_{m}\right)$ is bounded.

We define $B_{n m}(z ; \gamma)$ by

$$
\left(f^{\prime}, B_{n m}(z, \gamma) f\right)=\left(\left(\bar{\phi}_{n} \otimes f^{\prime}\right),\left(\bar{u}_{n}^{-1}(\gamma) \otimes 1\right) \beta_{n m}(\gamma)\left(\bar{u}_{m}(\gamma) \phi_{m} \otimes f\right)\right)
$$

In this case we have, for real $\gamma$

$$
\begin{aligned}
\left(f^{\prime},\right. & \left.u_{n}(\gamma) B_{n m}\left(z ; \gamma_{0}\right) u_{m}^{-1}(\gamma) f\right) \\
\quad= & \left(\left(\bar{\phi}_{n} \otimes f^{\prime}\right),\left(\bar{u}_{n}^{-1}\left(\gamma_{0}\right) \otimes u_{n}(\gamma)\right) \beta_{n m}\left(\gamma_{0}\right)\left(\bar{u}_{m}\left(\gamma_{0}\right) \otimes u_{m}^{-1}(\gamma)\right)\left(\phi_{m} \otimes f\right)\right) \\
\quad= & \left(\left(\bar{\phi}_{n} \otimes f^{\prime}\right),\left(\bar{u}_{n}^{-1}\left(\gamma_{0}\right) \bar{u}_{n}^{-1}(\gamma) \otimes 1\right) u(\gamma) \beta_{n m}\left(\gamma_{0}\right) u^{-1}(\gamma)\left(\bar{u}_{m}(\gamma) \bar{u}_{m}\left(\gamma_{0}\right) \otimes 1\right)\left(\phi_{m} \otimes f\right)\right) \\
\quad & \left(\left(\bar{\phi}_{n} \otimes f^{\prime}\right),\left(\bar{u}_{n}^{-1}\left(\gamma \times \gamma_{0}\right) \otimes 1\right) \beta_{n m}\left(\gamma \times \gamma_{0}\right)\left(\bar{u}_{m}\left(\gamma \times \gamma_{0}\right) \phi_{m} \otimes f\right)\right)
\end{aligned}
$$

\section{Extension to Distorted Contours}

We describe a family of distorted contours by a set of functions $q_{n}(s), n=1, \ldots, j$. Each function $q_{n}(s)$ maps $\mathbb{R}^{+}$into a curve $\Gamma_{n}$ in the complex plane. For $s \leqq s_{l}$ and $s \geqq s_{u}$ we require $q_{n}(s)=\lambda s$, where $\lambda$, complex, is the same for all $n$ for a given family, but may be different for another family. We assume that $q_{n}(s)$ is nine times differentiable and that

$$
\begin{aligned}
& J_{n}(s)=\frac{q_{n}^{2}}{s^{2}} \frac{d q_{n}}{d s} \neq 0, s \in \mathbb{R}^{+}, \text {and will place further restrictions on } q_{n}(s) \text { later. } \\
& \text { If } q_{n}(s) \text { is real, we may define a unitary operator } Q_{n}: L^{2}\left(\mathbb{R}^{3}\right) \rightarrow L^{2}\left(\mathbb{R}^{3}\right) \text { by } \\
& \left(Q_{n} f\right)(s \hat{\mathbf{s}})=J_{n}^{1 / 2}(s) f\left(q_{n}(s) \hat{\mathbf{s}}\right), \hat{\mathbf{s}} \in \mathbb{R}^{3},|\hat{\mathbf{s}}|=1 .
\end{aligned}
$$

If we allow $Q_{n}$ to act in the momentum representation of $\mathscr{H}_{n}^{(1,2)}$, we may define operators $B_{n m}^{q}(z)$ by

$$
B_{n m}^{q}(z)=Q_{n} B_{n m}(z) Q_{m}^{-1}
$$

Our task is to define a suitable extension of $B_{n m}^{q}(z)$ when $\left\{q_{n}(s)\right\}$ is complex. Formally, this will be achieved in the following manner. Suppose that in momentum space $B_{n m}(z ; \gamma)$ has the kernel $\left(\mathbf{p}^{\prime}\left|B_{n m}(z ; \gamma)\right| \mathbf{p}\right)$, using the Dirac notation. Then (9) is equivalent to

$$
\left(\zeta^{-1} \mathbf{p}^{\prime}+\bar{M} \tau_{n}\left|B_{n m}(z)\right| \zeta^{-1} \mathbf{p}+\bar{M} \tau_{m}\right)=\left(\mathbf{p}^{\prime}\left|B_{n m}(z ; \gamma)\right| \mathbf{p}\right)
$$


where $\tau_{m}$ is the projection of $\tau$ to the $\mathbb{R}^{3}$ corresponding to the relative motion of the clusters of $D_{m}^{2}$ (see [9]). To continue $B_{n m}^{q}(z)$ we need $\left(q_{n}\left(s^{\prime}\right) \hat{\mathbf{s}}^{\prime}\left|B_{n m}(z)\right| q_{m}(s) \hat{\mathbf{s}}\right)$ which may be defined from (11) by choosing $\gamma$ so that, with $\mathbf{p}=\mathbf{s}, \mathbf{p}^{\prime}=\mathbf{s}^{\prime}$

$$
\begin{aligned}
& \mathbf{q}_{m}(s)=q_{m}(s) \hat{\mathbf{s}}=\zeta^{-1} \mathbf{p}+\bar{M} \tau_{m} \\
& \mathbf{q}_{n}\left(s^{\prime}\right)=q_{n}\left(s^{\prime}\right) \hat{\mathbf{s}}^{\prime}=\zeta^{-1} \mathbf{p}^{\prime}+\bar{M} \tau_{n}
\end{aligned}
$$

In this case we have

$$
\begin{aligned}
\left(\mathbf{s}^{\prime}\left|B_{n m}^{q}(z)\right| \mathbf{s}\right) & =J_{n}^{1 / 2}\left(s^{\prime}\right)\left(\mathbf{q}_{n}\left(\mathbf{s}^{\prime}\right)\left|B_{n m}(z)\right| \mathbf{q}_{m}(\mathbf{s})\right) J_{m}^{1 / 2}(s) \\
& =J_{n}^{1 / 2}\left(s^{\prime}\right)\left(\mathbf{s}^{\prime}\left|B_{n m}(z, \gamma)\right| \mathbf{s}\right) J_{m}^{1 / 2}(s)
\end{aligned}
$$

To proceed rigorously, without assuming the existence of a kernel with suitable properties, we must first specify a solution of (12) for $\gamma$. For $n \neq m$, it is adequate to choose $\zeta^{-1}=\lambda 1$, and use (12) to define $\tau_{m}, \tau_{n}$.

In the case $n=m$, we define

$$
\zeta^{-1}=h_{1}\left(\left|\mathbf{s}-\mathbf{s}^{\prime}\right|\right) \mathscr{M}_{1}+h_{2}\left(\left|\mathbf{s}-\mathbf{s}^{\prime}\right|\right) \mathscr{M}_{2}
$$

where $h_{1}, h_{2}$ are six times differentiable and satisfy $h_{1}(x)+h_{2}(x)=1, h_{1}(x)=0$, $x<x_{l}, h_{1}(x)=1, x>x_{u}>x_{l}$. We choose

$$
\begin{aligned}
& \mathbf{a} \cdot \mathscr{M}_{1} \mathbf{b} \\
& =\frac{\left[\mathbf{a} \cdot\left(\mathbf{q}_{m}(\mathbf{s})-\mathbf{q}_{m}\left(\mathbf{s}^{\prime}\right)\right)\right]\left[\left(\mathbf{s}-\mathbf{s}^{\prime}\right) \cdot \mathbf{b}\right]-\left[\mathbf{a} \cdot\left(\mathbf{s}-\mathbf{s}^{\prime}\right)\right]\left[\left(\mathbf{s}-\mathbf{s}^{\prime}\right) \cdot \mathbf{b}\right] \lambda}{\left|\mathbf{s}-\mathbf{s}^{\prime}\right|^{2}}+\mathbf{a} \cdot \mathbf{b} \lambda
\end{aligned}
$$

and

$$
\left(\mathscr{M}_{2}\right)_{i j}=\left(\mathscr{M}_{3}\right)_{i j}+\frac{\rho_{i}\left(s_{j}-s_{j}^{\prime}\right)}{\left|\mathbf{s}-\mathbf{s}^{\prime}\right|^{2}},
$$

where

$$
\begin{aligned}
\left(\mathscr{M}_{3}\right)_{i j}= & \left.\sum_{n=1}^{6} \frac{1}{n !} \sum_{i_{1} \ldots i_{n-1}=1}^{3} \frac{\partial}{\partial s_{i_{1}}} \ldots \frac{\partial}{\partial s_{i_{n-1}}} \frac{\partial}{\partial s_{j}} q_{i}(\mathbf{s})\right|_{\mathbf{s}=\mathbf{s}^{\prime}} \\
& \left(s_{i_{1}}-s_{i_{1}}^{\prime}\right) \ldots\left(s_{i_{n-1}}-s_{i_{n-1}}^{\prime}\right)
\end{aligned}
$$

and

$$
\rho_{i}=q_{i}(\mathbf{s})-q_{i}\left(\mathbf{s}^{\prime}\right)-\sum_{j=1}^{3}\left(\mathscr{M}_{3}\right)_{i j}\left(s_{j}-s_{j}^{\prime}\right) .
$$

Again, we use (12) to define $\tau_{m}$.

We see that, if $q_{m}(s)$ and its derivatives are close enough to $\lambda s$ and its derivatives, then the corresponding $\gamma$ will belong to $S_{2}$.

To obtain a rigorous definition of $B_{n m}^{q}(z)$ for complex $q$, we express $B_{n m}\left(z_{i} \gamma\left(\mathbf{s}^{\prime}, \mathbf{s}\right)\right)$ appearing in (13) in terms of integrals of derivatives of $B_{n m}(z ; \gamma)$ with respect to $\gamma$. Let us define

$$
\begin{aligned}
\mathscr{P}^{1}: L^{2}(\mathbb{R}) \rightarrow L^{2}(\mathbb{R}) \text { by } \\
\left(\mathscr{P}^{1} f\right)(s)=f(s), \quad s \geqq 0 \\
0, \quad s<0
\end{aligned}
$$


and $\mathscr{P}^{2}=1-\mathscr{P}^{1}$. Then $B_{n m}^{q}(z)$ may be written

$$
B_{n m}^{q}(z)=\sum_{i_{1}=1}^{2} \ldots \sum_{i_{6}=1}^{2} \mathscr{P}^{i_{1}} \otimes \mathscr{P}^{i_{2}} \otimes \mathscr{P}^{i_{3}} B_{n m}^{q}(z) \mathscr{P}^{i_{4}} \otimes \mathscr{P}^{i_{5}} \otimes \mathscr{P P}^{i_{6}}
$$

We define the extension of $B_{n m}^{q}(z)$ by extending each of the $2^{6}$ terms in the expansion of (14) separately. We give the argument for $\mathscr{P}^{+} B_{n m}^{q}(z) \mathscr{P}^{+}$, where $\mathscr{P}^{+}=\mathscr{P}^{1} \otimes \mathscr{P}^{1} \otimes$ $\mathscr{P}^{1}$, and that for the other terms follows after a trivial modification.

We define $S_{i}, i=1, \ldots, 6$ by $\mathbf{S}=\left(\mathbf{s}^{\prime}, \mathbf{s}\right)$ and write $B\left(S_{1}, \ldots, S_{6}\right)$ for $B_{n m}\left(z ; \gamma\left(\mathbf{s}^{\prime}, \mathbf{s}\right)\right)$. With repeated use of the formula $\left(S_{i} \geqq 0\right)$

$$
f\left(S_{i}\right)=f(0)+\int_{0}^{S_{i}} d T_{i} \frac{\partial f}{\partial T_{i}}=f(0)+\int_{0}^{\infty} d T_{i} \theta\left(S_{i}-T_{i}\right) \frac{\partial f}{\partial T_{i}}
$$

We have an expression of the form

$$
\begin{aligned}
B\left(S_{1}, \ldots S_{6}\right)=\sum_{k=0} \sum_{p} p( & \int_{0}^{\infty} d T_{1} \theta\left(S_{1}-T_{1}\right) \ldots \\
& \left.\int_{0}^{\infty} d T_{k} \theta\left(S_{k}-T_{k}\right)\right) \frac{\partial^{k}}{\partial T_{1} \ldots \partial T_{k}} B\left(T_{1} \ldots T_{k} 0 \ldots 0\right)
\end{aligned}
$$

where $p$ runs over all choices of $k$ integers from $1, \ldots, 6$. The function $\theta\left(S_{1}-T_{1}\right)$ may be interpreted as a multiplicative bounded operator $\theta_{T_{1}}$. This leads us to our rigorous definition of $\mathscr{P}^{+} B_{n m}^{q} \mathscr{P}^{+}$which is

Definition 1. For $z \in S_{1}$ and $q$ complex so that the corresponding $\gamma$ all belong to $S_{2}$, we define $\mathscr{P}^{+} B_{n m}^{q}(z) \mathscr{P}^{+}$by

$$
\begin{aligned}
\mathscr{P}^{+} B_{n m}^{q}(z) \mathscr{P}^{+}=J^{1 / 2} \mathscr{P}^{+} \sum_{k=0}^{6} \sum_{p} p \int_{0}^{\infty} d T_{1} \ldots \int_{0}^{\infty} d T_{k} \\
\theta_{T_{1}} \ldots \frac{\partial^{k}}{\partial T_{1} \ldots \partial T_{k}} B\left(T_{1} \ldots T_{k} 0 \ldots 0\right) \ldots \theta_{T_{k}} \mathscr{P}^{+} J^{1 / 2}
\end{aligned}
$$

where $\theta_{T_{i}}$ is placed to the left or right of the operator depending whether $i$ is $\leqq 3$ or $>3$. For the other parts of $B_{n m}^{q}$, we replace $\int_{0}^{\infty} d T_{i} \theta\left(S_{i}-T_{i}\right)$ by $-\int_{-\infty}^{0} d T_{i}$ $\theta\left(T_{i}-S_{i}\right)$ where appropriate.

Remark 1. The convergence of the integrals in (15) follows since $\frac{\partial^{k}}{\partial T \ldots \partial T_{k}} B\left(T_{1} \ldots\right.$ $\left.T_{k} 0 \ldots 0\right)$ may be written as a sum of terms involving products of factors such as $G=\frac{\partial^{n} \gamma^{l}\left(\mathbf{t}^{\prime}, \mathbf{t}\right)}{\partial t_{i_{1}}^{\prime} \ldots \partial t_{i_{m}}^{\prime} \partial t_{i_{m+1}} \ldots \partial t_{i_{n}}}$ and $\frac{\partial B(z ; \gamma)}{\partial \gamma^{l_{1}} \ldots \partial \gamma^{l_{n}}}$, where $\gamma^{l}$ is an element of $\zeta^{-1}$ or $\tau$.

It follows from our definition of $\gamma\left(\mathbf{t}^{\prime}, \mathbf{t}\right)$ that $|G|<$ const $\mid\left(1+t_{i_{1}}^{\prime}\right) \ldots\left(1+t_{i_{m}}^{\prime}\right) \times$ $\left.\left(1+t_{i_{m+1}}\right) \ldots\left(1+t_{i_{n}}\right)\right|^{-8 / 7}$ and the derivatives of $B(z ; \gamma)$ with respect to $\gamma$ are uniformly bounded since, for all $\mathbf{t}, \mathbf{t}^{\prime}, \gamma\left(\mathbf{t}^{\prime}, \mathbf{t}\right)$ remains bounded.

Lemma 3. For $z \in S_{1}$ and $q$ real so that the corresponding $\gamma$ all belong to $S_{2}, B_{n m}^{q}(z)$ as given by Definition 1 coincides with $Q_{n} B_{n m} Q_{m}^{-1}$ of (10). 
Proof. We show that $\mathscr{P}^{+} B_{n m}^{q}(z) \mathscr{P}^{+}$of (15) equals $\mathscr{P}^{+} Q_{n} B_{n m} Q_{m}^{-1} \mathscr{P}^{+}$. Let $h_{n}(x)=$ $e^{-x^{2}} H_{n}(x), n=0, \ldots$, where $H_{n}(x)$ are normalized Hermite polynomials. Let $\chi_{i j k}(\mathbf{s})=h_{i}\left(s_{1}\right) h_{j}\left(s_{2}\right) h_{k}\left(s_{3}\right)$ and define the orthogonal projection $p_{i j k}$ by

$$
\left(p_{i j k} f\right)(\mathbf{s})=\chi_{i j k}(\mathbf{s})\left(\chi_{i j k}, f\right)
$$

Now set

$$
p_{N}=\sum_{\substack{i j k \\ i^{2}+j^{2}+k^{2}<N^{2}}} p_{i j k}
$$

Then we have $p_{N} B_{n m}(z) p_{N} \rightarrow B_{n m}(z)$ as $N \rightarrow \infty$, and similarly, for $\gamma$ real, $b_{N}(\gamma)=$ $u(\gamma) p_{N} B_{n m}(z) p_{N} u^{-1}(\gamma) \underset{s}{\rightarrow} u(\gamma) B_{n m}(z) u^{-1}(\gamma)=B_{n m}(z ; \gamma)$.

Now $b_{N}(\gamma)$ is an operator, analytic in $\gamma$, that satisfies the properties demonstrated for $B_{n m}(z ; \gamma)$ in Lemma 2. It is also represented by a kernel $\left(\mathbf{s}^{\prime}|b(\gamma)| \mathbf{s}\right)$, analytic in $\mathbf{s}, \mathbf{s}^{\prime}$ that obeys property (9). Thus the previous empirical argument applies rigorously in this case, and we have

$$
\begin{array}{r}
\mathscr{P}^{+} Q_{n} p_{N} B_{n m}(z) p_{N} Q_{m}^{-1} \mathscr{P}^{+}=J^{1 / 2} \mathscr{P}^{+} \sum_{k=0}^{6} \sum_{p} p \int_{0}^{\infty} d T_{1} \ldots \int_{0}^{\infty} d T_{k} \theta_{T_{1}} \ldots \frac{\partial^{k}}{\partial T_{1} \ldots \partial T_{k}} \\
{\left[b_{N}(\gamma(T O))\right] \ldots \theta_{T_{k}} \mathscr{P}^{+} J^{1 / 2}}
\end{array}
$$

The Lemma follows on taking $N \rightarrow \infty$.

In I the kernel of our integral equation $A_{n m}(z)$ was written as

$$
A_{n m}(z)=B_{n m}(z) \mathscr{K}_{m}(z)
$$

where $\mathscr{K}_{m}(z)=\omega^{-1} g_{m}(z) \omega^{-1}$. With the assumptions of I, we might just as well use the projection of $g_{m}$ onto a state of given angular momentum. Thus, from now on, we will replace $g_{m}(z)$ by $g_{m}^{l}(z)$, where $g_{m}^{l}(z)$ has momentum space kernel

$$
\left(\mathbf{p}^{\prime}\left|g_{m}^{l}(z)\right| \mathbf{p}\right)=Y_{l o}\left(\hat{\mathbf{p}}^{\prime}\right) Y_{l o}(\hat{\mathbf{p}})\left(z-b_{m}-p^{2} / 2 \mu_{m}\right)^{-1} \delta\left(p^{\prime}-p\right) p^{-2}
$$

As in the case of $B_{n m}(z)$ we may, for real $q_{m}$, define $\mathscr{K}_{m}^{q}(z)$ by

$$
\begin{aligned}
\mathscr{K}_{m}^{q}(z) & =Q_{m} \omega^{-1} g_{m}^{l}(z) \omega^{-1} Q_{m}^{-1} \\
& =\left(\omega^{-1}\right)^{q} Q_{m} g_{m}^{l}(z) Q_{m}^{-1}\left(\omega^{-1}\right)^{q}
\end{aligned}
$$

Since $\omega^{-1}$ is a bounded operator with the same transformation properties as $B_{n m}(z)$, we may define the extension $\left(\omega^{-1}\right)^{q}$ for complex $q$ as we did for $B_{n m}(z)$. We define the extension of $g_{m}^{l}(z)$ explicitly by

$$
\begin{aligned}
\left(\mathbf{s}^{\prime}\left|g_{m}^{l q}(z)\right| \mathbf{s}\right)= & J_{m}^{1 / 2}\left(s^{\prime}\right) Y_{l 0}\left(\hat{\mathbf{s}}^{\prime}\right) Y_{l 0}(\hat{\mathbf{s}})\left(z-b_{m}-q^{2}(s) / 2 \mu_{m}\right)^{-1} \\
& \cdot \delta\left(s^{\prime}-s\right) q^{-2}(s) J_{m}^{1 / 2}(s)
\end{aligned}
$$

so that for real $Q_{m}$

$$
g_{m}^{l q}(z)=Q_{m} g_{m}^{l}(z) Q_{m}^{-1} .
$$

Provided that $\bar{k}_{m}(z)=\left[2 \mu_{m}\left(z-b_{m}\right)\right]^{1 / 2}$ is not on $\Gamma_{m}, g_{m}^{l q}$ is obviously a bounded operator.

Lemma 4. For $\bar{k}_{m}(z) \notin \Gamma_{m}$ and the same restriction on $q$ as in Lemma $3, \mathscr{K}_{m}^{q}(z)$ is a 
Hilbert-Schmidt $(H-S)$ operator, which is also continuous in the H-S norm as $\bar{k}_{m}(z) \rightarrow k$.

Proof. We have $\omega^{-1}(\mathbf{r})=(\mathbf{r} \cdot \mathbf{r})^{-1 / 2}\left(1-e^{-(\mathbf{r} \cdot \mathbf{r}) 1 / 2}\right)$. Let the Fourier transform of $\omega^{-1}(\mathbf{r})$ be $W(\mathbf{p})$. Define the analytic family of bounded operators $\Omega(\zeta)$ by $(\Omega(\zeta) f(\mathbf{r})=$ $\omega^{-1}(\zeta \mathbf{r}) f(\mathbf{r})$. In momentum space $\Omega(\zeta)$ has the kernel

$$
\left(\mathbf{p}^{\prime}|\Omega(\zeta)| \mathbf{p}\right)=W\left(\zeta^{-1}\left(\mathbf{p}-\mathbf{p}^{\prime}\right)\right)(2 \pi)^{-3 / 2}(\operatorname{det} \zeta)^{-1}
$$

We write

$$
\left(\omega^{-1}\right)^{q}=\sum_{i_{1}=1}^{2} \sum_{i_{2}=1}^{2} \sum_{i_{3}=1}^{2} \mathscr{P}^{i_{1}} \otimes \mathscr{P}^{i_{2}} \otimes \mathscr{P}^{i_{3}}\left(\omega^{-1}\right)^{q}
$$

and show how to treat the term with $i_{1}=i_{2}=i_{3}=1$. The other terms are treated similarly.

As before, it may be shown that, for complex $q$ such that the corresponding $\gamma \in S_{2}$,

$$
\begin{aligned}
&\left(\mathbf{s}^{\prime}\left|\mathscr{P}^{+}\left(\omega^{-1}\right)^{q}\right| \mathbf{s}\right) \\
& \quad=\sum_{k=0}^{3} \sum_{p} p \int_{0}^{\infty} d T_{1} \ldots \int_{0}^{\infty} d T_{k} \theta_{T_{1}} \ldots \theta_{T_{k}}\left(s^{\prime}\left|\frac{\partial^{k}}{\partial T_{1} \ldots \partial T_{k}} \Omega\left(\zeta\left(T_{1} \ldots T_{k} 0 \ldots 0, \mathbf{s}\right)\right)\right| \mathbf{s}\right)
\end{aligned}
$$

where now $p$ chooses $k$ integers from 1,2, 3 .

We are thus led to study operators such as $X_{1} g_{m}^{l q}(z) X_{2}$ where $X_{1}, X_{2}$ have kernels

$$
\begin{aligned}
& \left(\mathbf{s}^{\prime}\left|X_{1}\right| \mathbf{s}\right)=\left(\mathbf{s}^{\prime}\left|\frac{\partial^{k}}{\partial \zeta^{l_{1}} \ldots \partial \zeta^{l_{n}}} \Omega\left(\zeta\left(T_{1} \ldots T_{k} 0 \ldots 0, \mathbf{s}\right)\right)\right| \mathbf{s}\right) \\
& \left(\mathbf{s}\left|X_{2}\right| \mathbf{s}^{\prime}\right)=\left(\mathbf{s}\left|\frac{\partial^{n}}{\partial \zeta^{l_{1}} \ldots \partial \zeta^{l_{n}}} \Omega\left(\zeta\left(\mathbf{s}, T_{1}^{\prime} \ldots T_{n}^{\prime} 0 \ldots 0\right)\right)\right| \mathbf{s}^{\prime}\right)
\end{aligned}
$$

In coordinate space $X_{1} g_{m}^{l q}(z) X_{2}$ has kernel

$$
\begin{gathered}
\left(\mathbf{r}^{\prime}\left|X_{1} g_{m}^{l q}(z) X_{2}\right| \mathbf{r}\right)=\mathrm{const} \int d \overline{\mathbf{s}}^{\prime} d \overline{\mathbf{s}} f_{1}\left(\mathbf{r}^{\prime}, \overline{\mathbf{s}}^{\prime}\right) e^{i \mathbf{r}^{\prime} \cdot \overline{\mathbf{s}}^{\prime}} \\
\left(\overline{\mathbf{s}}^{\prime}\left|g_{m}^{l q}(z)\right| \overline{\mathbf{s}}\right) f_{2}\left(\mathbf{r}^{\prime}, \overline{\mathbf{s}}\right) e^{-i \mathbf{r} \cdot \overline{\mathbf{s}}}
\end{gathered}
$$

where

$$
f_{1}\left(\mathbf{r}^{\prime}, \overline{\mathbf{s}}^{\prime}\right)=\frac{\partial^{k}}{\partial \zeta^{l_{1}} \ldots \partial \zeta^{\zeta}} \bar{l}_{k} \omega^{-1}\left(\zeta \mathbf{r}^{\prime}\right) \text { with } \zeta=\zeta\left(T_{1} \ldots T_{k} 0 \ldots 0, \overline{\mathbf{s}}^{\prime}\right)
$$

Using (16) we obtain

$$
\left(\mathbf{r}^{\prime}\left|X_{1} g_{m}^{l q}(z) X_{2}\right| \mathbf{r}\right)=\int_{0}^{\infty} d s s^{2} F_{1}\left(\mathbf{r}^{\prime}, s\right) F_{2}(\mathbf{r}, s)\left(z-b_{m}-q^{2}(s) / 2 \mu_{m}\right)^{-1}
$$

where

$$
F_{1}\left(\mathbf{r}^{\prime}, s\right)=\text { const } \int d \hat{\mathbf{s}} f_{1}\left(\mathbf{r}^{\prime}, \mathbf{s}\right) e^{i \mathbf{r}^{\prime} \cdot \mathbf{s}} Y_{l 0}(\hat{\mathbf{s}})
$$

and similarly for $F_{2}$. Straightforward estimates using the explicit form of $\omega^{-1}$ 
show that uniformly

$$
\left|F_{1}(\mathbf{r}, s)\right|<\operatorname{const}(r s)^{-1}(|\mathbf{r}|+1)^{-1}
$$

and

$$
\left|\frac{\partial F_{1}(\mathbf{r}, s)}{\partial s}\right|<\text { const } r^{-1}(|\mathbf{r}|+1)^{-1}
$$

and similarly for $F_{2}$. It follows that

$$
\left|\left(\mathbf{r}^{\prime}\left|X_{1} g_{m}^{l q}(z) X_{2}\right| \mathbf{r}\right)\right|<\mathrm{const}\left[r^{\prime}\left(\left|\mathbf{r}^{\prime}\right|+1\right) r(|\mathbf{r}|+1)\right]^{-1}
$$

even in the limit $\bar{k}_{m}(z) \rightarrow \Gamma_{m}$. Thus the operator $X_{1} g_{m}^{l q}(z) X_{2}$ is $H-S$.

We must also extend the inhomogeneous term of the integral equation I (6). Define $\chi\left(k_{n}\right) \in \mathscr{H}_{n}^{(1,2)}$ by

$$
\chi\left(k_{n}, \mathbf{r}\right)=\omega^{-1}(r) j_{l}\left(k_{n} r\right) P_{l}(\hat{\mathbf{r}})
$$

By explicit calculation from (8) $\chi\left(k_{n}\right)$ has momentum space representation

$$
\tilde{\chi}\left(k_{n}, \mathbf{p}\right)=\text { const }(p k)^{-1}\left[Q_{l}\left(\frac{p^{2}+k^{2}}{2 p k}\right)-Q_{l}\left(\frac{p^{2}+k^{2}+1}{2 p k}\right)\right]
$$

For complex $k_{n}$ and distorted contour $\Gamma_{n}$ with $k_{n} \in \Gamma_{n}$, we define the continuation $\chi^{q}\left(k_{n}\right)$ by

$$
\chi^{q}\left(k_{n}, \mathbf{s}\right)=J_{n}^{1 / 2}(s) \tilde{\chi}\left(k_{n}, q_{n}(s) \hat{\mathbf{s}}\right)
$$

It is easy to check that $\chi^{q}\left(k_{n}\right) \in L^{2}\left(\mathbb{R}^{3}\right)$ and that for real $k_{n}$ and $q_{n}(s), \chi^{q}\left(k_{n}\right)=Q_{n} \chi\left(k_{n}\right)$.

The function $\alpha_{n}(z)$ appearing in I(6) may be expressed as sums of products of operators with properties similar to $B_{n m}(z)$ and $\mathscr{K}_{m}(z)$ acting on $\chi\left(k_{i}\right)$. To extend $\alpha_{n}(z)$, we extend these operators as before and at the same time extend $\chi\left(k_{i}\right)$ to $\chi^{q}\left(k_{i}\right)$. In this way we obtain $\alpha_{n}^{q}\left(z ; k_{i}\right)$ defined for complex $k_{i} \in \Gamma_{i}$, with $z \in S_{1}$, each $\bar{k}_{m}(z)$ above the corresponding $\Gamma_{m}$ and $q$ chosen so that the corresponding $\gamma$ all belong to $S_{2}$, with the property that, for real $k_{i}, q, q_{n}^{q}\left(z ; k_{i}\right)=\alpha_{n}(z)$.

\section{Analyticity}

The aim of this section is to demonstrate that there exists a neighborhood of the segment $S_{3}=\left\{z: \operatorname{Im} z=0, b_{r}<\operatorname{Re} z<E_{0}\right\}$ of the real axis into which the physical scattering amplitude $T_{f i}(z)$ may be continued from real values of $z$. (The Born term may be dealt with easily).

Suppose that $z_{0} \in S_{1}$ with $\operatorname{Im} z_{0}<0$. To continue from real values of $z$ to $z_{0}$ we shall use various contour distortions $\left\{q_{n}(s)\right\}$ corresponding to $\Gamma_{n}$ in the fourth quardant, and we assert that $\left|\operatorname{Im} z_{0}\right|$ may be chosen small enough so that all the $q$ concerned satisfy the restrictions of Lemma 3. This assertion may be checked without difficulty and we shall assume its validity in the discussion below.

Let us write $k_{n}^{0}=\bar{k}_{n}\left(z_{0}\right)\left(\operatorname{Im} k_{n}^{0}<0\right)$, and suppose that $q^{0}=\left\{q^{0}(s)\right\}$ is such that $q_{f}^{0}(1)=k_{f}^{0}, q_{i}^{0}(1)=k_{i}^{0}$, and the contours $\Gamma_{n}^{0}(n \neq i$ or $f)$ pass below the corresponding points $k_{n}^{0}$. We shall assume for these contours that $\lambda=1$. It will also be assumed 
that there exist $\varepsilon_{n}>0$ such that for $1-\varepsilon_{n} \leqq s \leqq 1+\varepsilon_{n}, \operatorname{Im} q_{n}^{0}(s)=\operatorname{Im} k_{n}^{0}, n=i$ or $f$, and that $\left|\operatorname{Re} q_{n}^{0}\left(1+\varepsilon_{n}\right)-\operatorname{Re} k_{n}^{0}\right|>\delta_{n}>0$.

Now we define

$$
q_{n}\left(\theta_{n}, s\right)=\operatorname{Re} q_{n}^{0}(S)+\theta_{n} \operatorname{Im} q_{n}^{0}(s)
$$

We set

$$
\theta_{n}=\frac{k_{n}-\operatorname{Re} k_{n}^{0}}{\operatorname{Im} k_{n}^{0}}, n=i \text { or } f
$$

and leave the remaining $\theta_{n}$ as variables denoted collectively by $\theta$.

For $z \in S_{1}-S_{1}^{d}$ with $\operatorname{Im} z>0$ and suitable $k_{i}, k_{f}$ with negative imaginary parts we denote by $u_{n}$ the solution of

$$
u_{n}=q_{n}^{q}\left(z ; k_{i}\right)+\sum_{m=1}^{j} A_{n m}^{q}(z) u_{m}
$$

where $A_{n m}^{q}(z)=B_{n m}^{q}(z) \mathscr{K}_{m}^{q}(z)$, and we are using $q$ given by (17). Since the kernel of this equation is a $H-S$ operator, analytic in $z$, there will be a unique solution for all but a finite set $S_{q}^{d}$, and we suppose that $z \notin S_{q}^{d}$. We define the off-shell partial-wave scattering amplitude $T_{f i}\left(z ; k_{f}, k_{i}, \theta\right)$ by

$$
T_{f i}\left(z ; k_{f}, k_{i}, \theta\right)=\left(X^{q}\left(k_{f}\right)^{*}, u_{f}\right)
$$

The kernel of (18) has a Fredholm determinant $D\left(z ; k_{f}, k_{i}, \theta\right)$ whose zeroes in $S_{1}$ comprise $S_{q}^{d}$. We have

Lemma 5. Provided that the variables are chosen so that, for each $n, \vec{k}_{n}(z)$ is above the contour corresponding to (17), D depends only on $z$ (analytically for $z \notin S_{1}^{d}$ ).

Proof. The functions $q_{n}\left(\theta_{n}, s\right)$ depend analytically on $k_{f}, k_{i}, \theta$ for fixed $s$, and it follows from the definition of $A_{n m}^{q}(z)$ that this operator depends analytically on these variables. It is also analytic in $z$ for each $\bar{k}_{n}(z)$ above its corresponding contour. For $\operatorname{Im} z>0, D$ may be continued from $k_{f}, k_{i}=k_{f}^{0}, k_{i}^{0}, \theta=\{i\}$ to real values, each quantity varying over a segment of the real axis. In this case $D$ is independent of $k_{f}, k_{i}, \theta$, for $A_{n m}^{q}(z)$ is related to $A_{n m}(z)$ by a unitary transformation that leaves $D$ unchanged. The result follows by analytic continuation.

This lemma means that $S_{q}^{d}$ is independent of $k_{f}, k_{i}, \theta$ provided each $\bar{k}_{n}(z)$ is above its corresponding contour. We also see that we may construct an open disk $d$ with centre $z_{0}$, such that $D(z)$ is analytic, $z \in d+\partial d$. To do this it will be necessary to use in (18) $k_{n}=\operatorname{Re} k_{n}^{0}+i K_{n},\left|K_{n}\right|>\left|\operatorname{Im} k_{n}^{0}\right|$.

Lemma 6. The amplitude $T_{f i}\left(z ; k_{f}, k_{i}, \theta\right)$ is independent of $\theta$ and is analytic in $z, k_{f}, k_{i}, z \in S_{1}$, except possibly for poles at $z \in S_{1}^{d}+S_{q}^{d}$, provided that, for each $n, \bar{k}_{n}(z)$ is above its corresponding contour.

\section{Proof. As in Lemma 5.}

We shall call the amplitude $T_{f i}\left(z ; k_{f}, k_{i}\right)$.

Now let $\beta_{0}=\max _{z \in d}\left|\arg \bar{k}_{j}(z)\right|$. Suppose that $d$ has been chosen sufficiently small that we can find $\beta>\beta_{0}$ for which $d$ is contained in that part of $\left(\mathbb{C}-\sigma_{e}\left(0, e^{-i \beta} 1\right)\right)$ 
obtained by continuation to $\operatorname{Im} z<0$ through $\left(b_{j}, E_{\min }\right)$. We set

$$
k_{n}^{\beta}=\operatorname{Re} k_{n}^{0}(1-i \tan \beta), n=i \text { or } f .
$$

We suppose that $\left|\operatorname{Im} z_{0}\right|$ has been chosen small enough so that $T_{f i}\left(z ; k_{f}, k_{i}\right)$ may be continued along $\operatorname{Re} k_{f}=\operatorname{Re} k_{f}^{0}, \operatorname{Re} k_{i}=\operatorname{Re} k_{i}^{0}$ to a neighborhood of $z=z_{0}, k_{f}=$ $k_{f}^{\beta}, k_{i}=k_{i}^{\beta}$.

Next we define $F\left(z ; x_{f}, x_{i}\right), x_{f}, x_{i} \in \mathbb{C}$, by $F\left(z ; x_{f}, x_{i}\right)=T_{f i}\left(z ; x_{f}+\bar{k}_{f}(z)\right.$, $\left.x_{i}+\bar{k}_{i}(z)\right)$. We have

Lemma 7. For sufficiently small d there exist $\delta_{f}^{\prime}, \delta_{i}^{\prime}, h_{f}, h_{i}>0$ such that $F\left(z ; x_{f}, x_{i}\right)$ is meromorphic in the polycylinder $\left|\operatorname{Re} x_{n}\right|<\delta_{n}^{\prime},-h_{n}<\operatorname{Im} x_{n}<0, n=i$ or $f$, $z \in d+\partial d$. Poles of $F\left(z ; x_{f}, x_{i}\right)$ may occur only at those values of $z \in d+\partial d$ for which $H\left(0, e^{-i \beta} 1\right)$ has an eigenvalue.

Proof. The meromorphy is immediate from that of $T_{f i}\left(z ; k_{f}, k_{i}\right)$, for provided that $\left|\operatorname{Re}\left(k_{n}-\bar{k}_{n}(z)\right)\right|<\delta_{n}, n=i$ or $f$, our choice of $\{q\}$ ensures that $\bar{k}_{n}(z)$ will lie above the corresponding contour if $\operatorname{Im}\left(k_{n}-\bar{k}_{n}(z)\right)<0, n=i$ or $f$, and $z \in d+\partial d, d$ sufficiently small.

To demonstrate the relation of poles of $F$ with eigenvalues of $H\left(0, e^{-i \beta} 1\right)$, we define for $\operatorname{Im} z>0, \lambda, q>0$

$$
\begin{gathered}
T(z ; \lambda, q)=q^{3(N-1) / 2} \lambda^{-3(N-1)}\left(\psi_{f}(1),\left(1 \otimes \bar{u}_{f}(0, \lambda 1)\right) u\left(0, \lambda^{-1} 1\right)(z-H)^{-1}\right. \\
\left.u^{-1}\left(0, \lambda^{-1} 1\right)\left(u_{i}(0, q 1) \otimes \bar{u}_{i}\left(0, \lambda^{-1} 1\right)\right) \psi_{i}(1)\right)
\end{gathered}
$$

where $\psi_{i}(1)$ means the $\psi_{i}$ defined in I with $k_{i}=1$.

As explained in Sect. 2, this expression is analytic in $z, \lambda$ for $\arg \bar{k}_{j}(z)>\arg \lambda$, except possibly for poles in $z$ at the eigenvalues of $u\left(0, \lambda^{-1} 1\right) H u^{-1}\left(0, \lambda^{-1} 1\right)$.

For $\operatorname{Im} z>0$ and $\lambda, q$ real with $\lambda$ near $\operatorname{Re} k_{f}^{0}, \lambda q$ near $\operatorname{Re} k_{i}^{0}$, we have

$$
T(z ; \lambda, q)=T_{f i}(z ; \lambda, \lambda q) \text {. }
$$

Now suppose that the only point of $\left(S_{q}^{d}+S_{1}^{d}\right)$ in $z \in d+\partial d$ is at $z=z_{0}$, and that $H\left(0, e^{-i \beta} 1\right)$ has no eigenvalue at $z=z_{0}$ (and therefore none for $z \in d+\partial d$ ). By analytic continuation in $\lambda$ of (20) along a path near $\operatorname{Re} \lambda=\operatorname{Re} k_{f}^{0}$ with $q$ fixed near $\operatorname{Re} k_{i}^{0} / \operatorname{Re} k_{f}^{0}$, we deduce that $T_{f i}(z ; \lambda, \lambda q)$ is analytic in $z, \lambda$ for $z \in d+\partial d, q$ real and $\lambda, \lambda q$ in neighborhoods of $k_{f}^{\beta}, k_{i}^{\beta}$. We therefore have for such $\lambda, q$

$$
T_{f i}(z ; \lambda, \lambda q)=\frac{1}{2 \pi i} \int_{\partial d} d z^{\prime} \frac{T_{f i}\left(z^{\prime} ; \lambda, \lambda q\right)}{z^{\prime}-z}, z \in d .
$$

The right-hand side is analytic in $q, \lambda, z$, and so by analytic continuation is the left-hand side. It follows that $\delta_{n}^{\prime \prime}>0$ and the specifications of the polycylinder may be chosen so that $F\left(z ; x_{f}, x_{i}\right)$ is analytic for $\left|\operatorname{Re} x_{n}\right| \leqq \delta_{n}^{\prime},-h_{n}<\operatorname{Im} x_{n}<-h_{n}+$ $\delta_{n}^{\prime \prime}, n=i$ or $f, z \in d+\partial d$. Analytic continuation from this region of

$$
F\left(z ; x_{f}, x_{i}\right)=\frac{1}{2 \pi i} \int_{\partial d} d z^{\prime} \frac{F\left(z^{\prime} ; x_{f}, x_{i}\right)}{z^{\prime}-z}
$$

shows that $F\left(z ; x_{f}, x_{i}\right)$ is analytic throughout the polycylinder. 
If $H\left(0, e^{-i \beta} 1\right)$ has an eigenvalue at $z=z_{0}$ such that $\left(z-H\left(0, e^{-i \beta} 1\right)\right)^{-1}$ has a pole of order $v$ at this point, the above argument is easily modified to show that $\left(z-z_{0}\right)^{v} F\left(z ; x_{f}, x_{i}\right)$ is analytic inside the polycylinder.

We are now in a position to prove

Theorem 1. Subject to conditions (i) to (iii), the partial-wave on-shell scattering amplitude $\bar{T}_{f i}(z)=$ Born term $+T_{f i}(z)$ is meromorphic in a neighborhood of any segment of the real axis below $E_{\min }$ not containing thresholds, where physical scattering is possible. It may have poles only at eigenvalues of $H\left(0, e^{-i \beta} 1\right)$ which may be reached from this part of the real axis without crossing the continuous spectrum of this operator.

Proof. Suppose as in the previous Lemma that $H\left(0, e^{-i \beta} 1\right)$ has no eigenvalue for $z \in d+\partial d$. For $z \in d+\partial d$, a straightforward extension of Lemma 4 shows that uniformly

$$
\lim _{\varepsilon \downarrow 0} F(z ;-i \varepsilon,-i \varepsilon=F(z)
$$

Taking this limit in (21) shows that $F(z)$ is analytic for $z \in d$. If $H\left(0, e^{-i \beta} 1\right)$ has an eigenvalue, index $v$, at $z=z_{0}$, and no other for $z \in d+\partial d$, then we can show that $\left(z-z_{0}\right)^{v} F(z)$ is analytic for $z \in d$.

Covering a neighborhood of $S_{3}$ with disks such as $d$ shows that $F(z)$ is meromorphic in this neighborhood. For $z$ real, $z \in S_{3}$, we have $F(z)=T_{f i}(z)$ and the theorem follows by analytic continuation.

\section{References}

1. Nuttall, J., Singh, S. R. : Can. J. Phys. 57, 449-456 (1979)

2. Combes, J. M. : In: Atomic scattering theory, p. 25, (ed. J. Nuttall). University of Western Ontario, 1978

3. Tip. A. : In : Atomic scattering theory, p. 195, (ed. J. Nuttall) University of Western Ontario, 1978

4. Hagedorn, G. : A link between scattering resonances and dilation analytic resonances in few body quantum mechanics. Preprint, Rockefeller University (1978)

5. Nuttall, J. : J. Math. Phys. 8, 873-877 (1967)

6. Nuttall, J. : Comput. Phys. Commun. 6, 331-335 (1974)

7. Singh, S. R., Nuttall, J. : In : Atomic scattering theory, p. 185, (ed. J. Nuttall) University of Western Ontario, 1978

8. See for example Int. J. Quantum Chem. Vol. 14, (1978)

9. Combes, J. M., Thomas, L. : Commun. Math. Phys. 34, 251-270 (1973)

Communicated by J. Ginibre

Received February 27, 1979; in revised form August 10, 1979 
